\section{Porfiria. Diagnóstico diferencial en dolor abdominal}

\section{Sr. Director:}

Las porfirias, son enfermedades metabólicas poco frecuentes, causadas por la hipoactividad en algunas de las enzimas que forman parte de la síntesis del grupo hem.

Se describen 7 tipos de porfirias en función del fallo enzimático, presentándose clínicamente algunas de ellas como crisis agudas abdominopsiconeurológicas, en las que el dolor abdominal suele ser el síntoma de presentación.

La Porfiria Aguda Intermitente (PAI) es una variedad de porfiria, que tiene su defecto enzimático en la porfobilinogeno desaminasa. La prevalencia de la enfermedad es muy difícil de valorar, ya que la mayoría de los portadores del gen profírico son asintomático. En España el numero de casos diagnosticados es escaso, variando según los autores, aunque probablemente aumentarían, si se valorara la posibilidad de PAI en el diagnóstico diferencial de muy diversos procesos de difícil filiación. El diagnóstico de las crisis porfíricas agudas esta basado en la cuantificación en orina de ácido delta aminolevulinico (ALA), porfibilinogeno (PBG) y uroporfirinas.

Mujer de 25 años, con antecedentes de estreñimiento y dismenorrea, sin hábitos tóxicos ni alergias medicamentosas conocidas, que acude a nuestro Servicio de Urgencias por presentar dolor abdominal de carácter intermitente de 3 días de evolución, de inicio en hemiabdomen superior, que en las ultimas horas se ha hecho continuo y se localiza en fosas renales y fosas iliacas. Se acompaña de estreñimiento de 4 días de evolución.

No presenta fiebre, vómitos ni sintomatología urinaria. En la exploración destaca la ausencia de signos patológicos, excepto timpanismo abdominal en marco cólico. Se realizan estudios complementarios de urgencias: hemograma, bioquímica, y analítica de orina dentro de la normalidad, test gestación negativo, Rx simple de abdomen que muestra distensión de asas sin niveles hidroaereos y en la ecografía abdominal se evidencia colección de liquido en FID que sugería la posibilidad de patología apendicular por lo que se solicita valoración por cirujano.

Ante la persistencia de los síntomas y las nuevas pruebas complementarias con similares resultados, el cirujano decide la apendicectomía, encontrándose un apéndice normal. En los 3 días siguientes el dolor se intensifica, adquiriendo carácter cólico, lle- gando a precisar analgesia con opiáceos. Se realizan nuevos estudios complementarios: Rx de abdomen con distensión de asas y niveles hidroaéreos periumbilicales, Urograma normal, TAC abdominal con distensión de asas, hemograma sin leucocitosis pero con neutrofilia ( $82 \%$ segmentados), analíticas de orina con hematíes aislados, 1-2 leucocitos/campo y ligera bacteriuria.

El deterioro progresivo del estado general, junto con la intensidad creciente del dolor abdominal, lleva de nuevo a la paciente a quirófano para laparotomía exploradora que resulto ser negativa. La aparición de otros síntomas como taquicardia, elevación de cifras de tensión arterial, ansiedad, insomnio y alteraciones electrolíticas, junto con el dolor abdominal, nos lleva a la sospecha clínica de PAI, realizando de forma inmediata la pruebe empírica de exposición solar de una muestra de orina, siendo esta positiva (coloración intensa en unos 30 minutos). Con posterioridad se solicitan estudios en orina de $24 \mathrm{~h}$ de ALA 47,6 mg/24 (valor normal $1-7 \mathrm{mg} / 24 \mathrm{~h}$ ), PBG $45 \mathrm{mg} / 24 \mathrm{~h}$ (valor normal menor de 2 $\mathrm{mg} / 24 \mathrm{~h}$ ), uroporfirinas $2321 \mathrm{mg} / 24 \mathrm{~h}$ (valor normal menor de 60 $\mathrm{mg} / 24 \mathrm{~h}$ ), y estudios en heces de Coproprfirinas y Protoporfirinas con resultados normales. De esta forma quedo establecido el diagnostico definitivo de PAI, solicitándose a Sanidad Exterior el tratamiento específico consistente en argininato de Hemina. Durante los 3 días de espera hasta la llegada del medicamento, la paciente empeora, apareciendo alteraciones neurológicas severas (parestesias en extremidades, disminución de fuerza en miembros inferiores, disminución del nivel de conciencia y desconexión del medio), que se acompañan de una autentica catástrofe electrolítica por síndrome de secreción inadecuada de ADH (SIADH), con hiponatremia, hipokaliemia, hipocalcemia, hipofosfatemia, e hipomagnesemia, que precisa ingreso en la UCI para control.

Se realiza tratamiento con argininato de Hemina a razón de 3 $\mathrm{mg} / \mathrm{kg} /$ día (175 mg/día) durante 3 días, tras lo cual la paciente evoluciona muy favorablemente, desapareciendo los síntomas y normalizándose los trastornos electrolíticos. Es dada de alta de la UCI al $5^{\circ}$ día, y a su domicilio tras 7 días de estancia en planta.

La PAI es un defecto autosómico dominante de la biosíntesis del grupo HEM cuyo origen está en una reducción de la actividad de la enzima porfobilinógeno desaminasa (1), estando el defecto genético localizado en el cromosoma 11 (2). Su prevalencia en España es muy difícil de valorar, entre $1-8 / 100.000$ ya que la mayoría de los portadores del gen profírico son asintomáticos. Dicha cifra probablemente aumentaría si se considerase la posibilidad de PAI en el diagnóstico diferencial de muy diversos procesos de difícil filiación (3). 
El dolor abdominal es el síntoma inicial en el $85 \%$ de los pacientes con PAI, la afectación del sistema nervioso autónomo es la responsable de los espasmos y dilatación de asas intestinales; el estreñimiento, nauseas y vómitos son frecuentes. Así, es frecuente que sea catalogado, en un principio, como cuadro abdominal quirúrgico que ha llevado a no pocos enfermos de forma innecesaria al quirófano. Otros síntomas habituales son: taquicardia, ansiedad, confusión, afectación neuropática periférica motora y sensorial y convulsiones (4). Algunos pacientes pueden sufrir parálisis fláccida residual y en casos extremos parálisis respiratoria, coma y muerte (5). Entre otras manifestaciones, podemos destacar las alteraciones electrolíticas severas como hiponatremia, secundaria a SIADH, que pueden agravar de forma significativa la evolución de la crisis.

En el tratamiento específico de los ataques porfíricos, son dos las opciones más extendidas; por un lado la escuela americana que se inclina más por el uso de Hematina (6) y por otro, la opción europea que aconseja el uso de argininato de Hemina. Nosotros empleamos esta segunda posibilidad por su mayor estabilidad (7).

De entre todas las recomendaciones, el diagnóstico precoz debemos considerarlo como la herramienta ideal (8). De esta forma, en países como Suecia y debida a su alta prevalencia (9) se realizan preferentemente antes de la pubertad análisis genéticos en familias afectadas. Otra de las ventajas de dicho diagnóstico es la reducción en la yatrogenia derivada de actos quirúrgicos innecesarios.

Entre los factores desencadenantes de las crisis agudas de porfiria, hemos de mencionar con especial énfasis un alto número de fármacos, ayuno prolongado, dietas hipocalóricas, y fases luteínicas del ciclo menstrual. En algunas mujeres, la alta recidiva de crisis premenstruales hace recomendable el uso de análogos de LH-RH para evitar las crisis (10) (11). Mención aparte hay que hacer sobre el embarazo, ya que los ataques porfíricos pueden suceder durante este periodo, siendo potencialmente toxico el ALA por cruzar la barrera placentaria y dañar el normal desarrollo del cerebro fetal, por lo que se recomienda evitar el embarazo en los 2 años siguientes al ataque $(3,11)$.

\section{A. Gómez José, J. Campos Blanco, J. L. Pérez Fijo, M. J. Pérez Galisteo, F. Rabadán Bujalance}

Servicio de Urgencias y Cuidados Críticos. Hospital Victoria Eugenia (Cruz Roja Española). Sevilla

1. Frère T, Roy Peaud F, Ripault MP, Dumas P, Silvain C, Pourrat O, et al. Acute intermittent Porphyria associated whit hyperaldosteronysm and inappropriate antidiuretic hormone secretion syndrome. Gastroenterol Clin Biol 1998; 22 (8-9): 721-31.

2. Sedlák T, Pontuch P, Duris I. Acute intermittent Porphyria. Bratisl Lek Listy 1998; 99 (10): 536-7.

3. Enríquez de Salamanca R. Porfirias. En: Rozman C,Farreras P Directores. Medicina Interna. 13 ${ }^{\mathrm{a}}$ ed. Madrid: Mosby-Doyma Libros, 1995; vol. II. p. 1877-85.

4. Abinzano ML, Elejalde JL, Velilla JP, Alonso JL, Rubio T, García C. Porfiria aguda intermitente con manifestaciones neurológicas. An Med Interna (Madrid) 1999; 16: 66-67.

5. Paslin, DA. The porphyrias. In: Conn R, Borer W, Snyder J Editors. Current diagnosis 10. Philadelphia: WB Saunders Co., 1996. p. 717-26.

6. Morales Ortega X, Wolff Fernández C, Leal Ibarra T, Montaña Navarro N, Armas Merino R. Porphyric crisis: experience of 30 episodes. Medicina (Buenos Aires) 1999; 59 (1): 23-7.

7. Tenhunen R, Mustajoki P. Acute Porphyria: treatment with heme. Semin Liver Dis 1998; 18 (1): 53-5.

8. Bustamante M, Moll JL, Sarrion JV, Berenguer J. Acute intermittent Porphyria: a posible cause of abdominal pain. Gastroenterol Hepatol, 1999; 2 (10): 497-500.

9. Floderus Y, Harper P, Henrichson A, Thunell S, Anderson D. Preven- tion of acute intermittent Porphyria is the best solution. Most important are early diagnosis and counseling. Lakartidningen, 1998; 95 (26-27): 3045-50.

10. De Block CE, Leew IH, Gaal LF. Premenstrual attacks of acute intermittent Porphyria: hormonal and metabolic aspects - a case report. Eur J Endocrinol, 1999; 141 (1): 50-4.

11. Thadani H, Deacon A, Peters T. Diagnosis and management of Porphyria. BMJ 2000; 320:1647-51.

Disnea y anemia en relación con herniación de estómago y ángulo esplénico de colon a través del hiato esofágico

\section{Sr. Director:}

La gran mayoría de las hernias diafragmáticas se producen por deslizamiento del estómago a través del hiato esofágico. Otras hernias diafragmáticas menos comunes pero de mayor gravedad son las hiatales paraesofágicas, las congénitas del diafragma y las hernias diafragmáticas postraumáticas $(1,2)$.

Las hernias hiatales mixtas y paraesofágicas pueden ser asintomáticas o presentarse con manifestaciones abdominales inespecíficas como malestar o sensación de plenitud postprandiales (1). En ocasiones, sin embargo, son la causa de síntomas o signos atípicos, como disnea y anemia, que pueden dificultar el diagnóstico.

Varón de 67 años de edad, con antecedentes de hernia de hiato y anemia diagnosticada hace 2 años, que ingresa para estudio por disnea de años de evolución más evidente durante los últimos meses. La sintomatología se incrementa con la flexión dorsal y se acompaña de sensación de opresión precordial de características atípicas, intermitente, que se agrava con el esfuerzo y alivia al eructar, ventosear y/o defecar.

La exploración física no reveló ningún dato significativo. En la analítica destacaba una anemia microcítica e hipocroma de características ferropénicas. La Rx de tórax puso de manifiesto una imagen redondeada en mediastino posterior, con un nivel hidroaéreo en su interior.

Con la sospecha de hernia de hiato se realiza Gastroscopia en la que se observa una gran hernia de hiato paraesofágica, con posible volvulación del cuerpo gástrico. Estos hallazgos se confirman mediante estudio radiológico esófago-gastro-duodenal que objetiva un estómago intratorácico en posición invertida, con buena evacuación del contraste hacia el bulbo duodenal.

Como parte del estudio de anemia se realiza Enema opaco (Fig. 1) que demuestra herniación del ángulo esplénico del colon a través de la zona posteromedial del diafragma. La TAC puso de manifiesto que el orificio herniario en el diafragma correspondía al hiato esofágico.

Se propuso al paciente intervención quirúrgica como solución definitiva del problema, pero fue rechazada por el mismo.

La herniación del colon a través del hiato esofágico es un hallazgo inusual y suele estar asociado a la herniación parcial o total gástrica en disposición habitualmente paraesofágica y frecuentemente volvulado (3-6).

Las hernias diafragmáticas se definen como el desplazamiento de los órganos abdominales a la cavidad torácica a través de los orificios existentes en el diafragma $(1,2)$. Se clasifican, fundamentalmente, en dos tipos: congénitas o embrionarias, siendo la más común la hernia diafragmática posterolateral de Bochdalek; y adquiridas (hernias de Morgagni, hiatales y traumáticas) (2).

Las hernias hiatales son las más frecuentes del diafragma y 


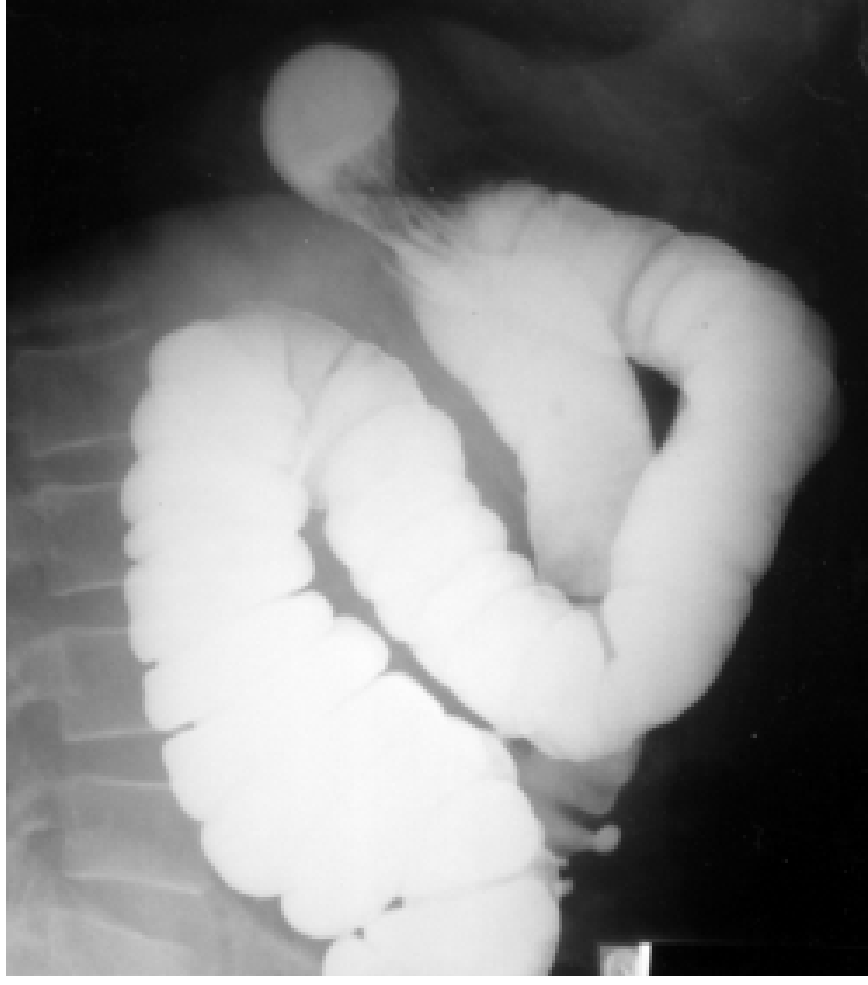

Fig. 1. Enema opaco (visión lateral) en la que se objetiva el ángulo esplénico del colon en posición intratorácica.

una de las alteraciones más comunes del tracto gastrotintestinal superior con una prevalencia de 5 casos por 1000 habitantes. Su incidencia crece paralelamente a la edad del paciente, siendo máxima entre los 40-70 años, y en el sexo femenino (75\% en algunas series) $(1,2)$.

La alteración de los mecanismos de contención hiatales pueden dar lugar a varios tipos de hernias: tipo I (por deslizamiento); tipo II (paraesofágica), la unión esofagogástrica se encuentra en su posición normal, fijada por la membrana frenoesofágica, pero el estómago se desliza hacia el tórax a través del hiato esofágico; tipo III (mixta o compuesta); y tipo IV (complicada), aquella que presenta un gran saco que permite el paso de otros órganos como colon, bazo, páncreas o intestino delgado, como en el caso que describimos.

Las hernias paraesofágicas tipo II pueden ser totalmente asintomáticas a pesar de su gran tamaño. Los síntomas se relacionan normalmente con el grado de obstrucción gástrica parcial o completa por la tendencia del estómago herniado a desarrollar vólvulo. La afectación de estómago y colon son más frecuentes en las grandes hernias paraesofágicas y excepcional en las formas por deslizamiento (3). El 80\% presentan dolor postprandial. También pueden ocurrir disnea, neumonía recurrente por aspiración, hemorragia por gastritis y anemia crónica. Nuestro paciente presentaba síntomas de disnea y sensación de opresión resultado del incremento de la presión intratorácica, así mismo la anemia podría ser explicada por las numerosas complicaciones a las que pueden ser sometidas las estructuras herniadas (1).

Este tipo de hernias tienen indicación quirúrgica ya que tienden a aumentar de tamaño y nunca regresan y debido a que la frecuencia de complicaciones se cifra en el $25 \%(7,8)$. La recurrencia a largo plazo tras la cirugía es del $15 \%$ (2), aunque en algunas series recientes la recidiva ha sido nula (8). La vía laparoscópica se ha ensayado también en caso de hernias no complicadas con buenos resultados (9). Debido a que la morbimortalidad de la cirugía de urgencia en caso de complicaciones (incarceración, hemorragia digestiva alta o insuficiencia respiratoria con disnea severa) es alta, sobre todo en pacientes ancianos, se recomienda la cirugía electiva en casos sintomáticos $(3,4)$. Sin embargo, también se ha ensayado tratamientos conservadores en algunas series con buenos resultados en caso de hernias no postraumáticas (6).

F.G. Benito Sánchez, J.A. Carneros Martín, C. Jiménez Pérez, M. Martínez Alonso, J. Torres Salcines, S. de la Torre Gutiérrez

Servicio de Aparato Digestivo. Hospital Militar Central Gómez Ulla. Madrid

1. Harford WV, McArthur KE. Divertículos, hernias, vólvulos y ruptura. En: Sleisenger MH, Fordtran JS. Eds. Enfermedades Gastrointestinales. Filadelfia: W.B. Saunders, 1993; 486-494.

2. Serrano Sánchez PA, Martín Pérez E. Hernias diafragmáticas. En: Rodés J., Malagelada JR, Pajares JM, Pérez Mota A, Moreno González E, Puig la Calle J. Eds. Enfermedades digestivas. Grupo Aula Médica 1998; $389-$ 309.

3. Fernández J, Gómez A, Arrizabalaga S, Gómez J, Taibo MA, Conty JL. Hernia transhiatal del estómago y colon transverso. Rev Esp Enf Digest 1992; 82: 371-372.

4. Wichterman K, Geha AS, Cahow CE, Baue AE. Giant paraesophageal hiatus hernia with intrathoracic stomach and colon: the case for early repair. Surgery 1979; 86: 497-506.

5. Farrell B, Gerad PS, Bryk D. Paraesophageal hernia causing colonic obstruction. J Clin Gastroenterol 1991; 13: 188-190.

6. Jewell FM, Tesar PAJ, Virjee J. Diaphragmatic herniation of the large bowel. Clinical Radiology 1994; 49: 469-472.

7. Leese T, Perdikis G. Management of patients with giant paraesophageal hernia. Dis Esophagus 1998; 11: 177-180.

8. Geha AS, Massad MG, Snow NJ, Baue AE. A 32-year experience in 100 patients with giant paraesophageal hernia: the case for abdominal approach and selective antireflux repair. Surgery 2000; 128: 623-630.

9. Wiechmann RJ, Ferguson MK, Naunheim KS, McKesey P, Hazelrigg SJ, Santucci TS, et al. Laparoscopic management of giant paraesophageal herniation. Ann Thorac Surg 2001; 71: 1080-1086.

\section{Taponamiento cardiaco como manifestación inicial} de un lupus eritematoso sistémico de comienzo tardío

\section{Sr. Director:}

La pericarditis se considera la manifestación cardiovascular más frecuente en el lupus eritematoso sistémico (LES). Sin embargo, no es habitual su progresión hasta producir compromiso hemodinámico y menos aún en el contexto del cuadro clínico de debut de la enfermedad $(1,2)$. Presentamos un caso con dos singularidades adicionales: su edad avanzada y la presencia de hipertensión pulmonar (HTP) aguda de origen no aclarado.

Mujer de 66 años con antecedentes de alergia a penicilina, espondiloartrosis, histerectomía por miomas, mastectomía izquierda con radioterapia coadyuvante por carcinoma de mama 27 años antes y síndrome depresivo diagnosticado seis meses atrás. Acudió a Urgencias por un cuadro de cuatro meses de evolución de dolores torácicos de localización cambiante, más intensos con la inspiración y al tumbarse sobre el lado doloroso, junto 
con pérdida de apetito y de $5 \mathrm{~kg}$ de peso, así como de sensación de dificultad respiratoria en el último mes. Negaba fiebre, escalofríos, tos, palpitaciones, síncope, ortopnea ni disnea paroxística nocturna. Asimismo, durante el último año refería episodios recurrentes de inflamación y dolor en ambas manos. La paciente estaba taquipneica, la auscultación cardiaca era rítmica y sin soplos ni roces, presentaba crepitantes en la base pulmonar derecha y ligeros edemas en ambas piernas. El resto de la exploración era normal.

Dentro de las pruebas complementarias destacaban 3.000 leucocitos (71\% neutrófilos y $21 \%$ linfocitos), hemoglobina de 10,1 $\mathrm{g} / \mathrm{dl}$ con VCM de $83 \mathrm{fl}$, un electrocardiograma en ritmo sinusal a $90 \mathrm{lpm}$ con hemibloqueo anterior, una $\mathrm{PO}_{2}$ de $69 \mathrm{mmHg}$ y $\mathrm{PCO}_{2}$ de $29,1 \mathrm{mmHg}$, una radiografía de tórax con un pequeño derrame pleural derecho y un dímero D-D levemente elevado con técnica de aglutinación con látex. Ante la posibilidad de embolia pulmonar, la paciente ingresa bajo anticoagulación. En planta se realizó gammagrafía pulmonar, TAC torácico helicoidal y estudio ecodoppler de miembros inferiores, sin encontrar datos concluyentes de enfermedad tromboembólica venosa. Sin embargo, en un ecocardiograma en el cuarto día de ingreso se estimó una presión sistólica pulmonar de $67,3 \mathrm{mmHg}$. Los días sucesivos presentó además picos febriles intermitentes de más de $38^{\circ} \mathrm{C}$, con cultivos negativos en todas las muestras obtenidas. Coincidiendo con un empeoramiento de la disnea, se realiza una nueva radiografía de tórax, detectándose un claro aumento de la silueta cardiaca. Tras revelar el ecocardiograma un derrame pericárdico severo con variabilidad del flujo mitral del $26 \%$ y presencia de pulso paradójico, se realizó una pericardiocentesis, obteniéndose $400 \mathrm{ml}$ de líquido hemorrágico con citología benigna y negatividad en el cultivo. En un ecocardiograma posterior se apreciaba un mínimo derrame residual y la ausencia de HTP. En los análisis realizados más tarde se observó un factor reumatoide y anticuerpos anticardiolipina negativos, siendo positivos los anticuerpos antinucleares (ANA) y los anti-ADN, a títulos de $1 / 1280$ y 325 respectivamente. El fenómeno LE en sangre fue también positivo.

Se estableció el diagnóstico de LES ante la presencia de leucopenia y linfopenia mantenidas, pleuropericarditis y positividad para ANA y anticuerpos anti-ADN. Tras la administración de prednisona a dosis de $1 \mathrm{mg} / \mathrm{kg} /$ día la paciente experimentó una progresiva mejoría clínica y radiológica al igual que la desaparición de su cuadro febril.

Se estima que más de la mitad de los pacientes con LES desarrollan derrame pericárdico a lo largo de su evolución (3), siendo aún más frecuente la afectación pericárdica si se consideran series necrópsicas. En una revisión de Moder y cols. (4) en la que se recogían 595 casos con evidencia clínica o ecográfica de pericarditis, sólo en 20 se objetivó taponamiento cardiaco, siendo infrecuente dentro de la clínica de presentación. En la mayoría de los pacientes se encuentra simultáneamente un derrame pleural, en el contexto de una serositis generalizada. Cuando se realiza pericardiocentesis, es inusual encontrar un líquido hemorrágico, si bien en nuestra paciente pudo influir de forma determinante el tratamiento anticoagulante al que fue sometida.

Se han descrito varios procesos asociados a HTP en pacientes con LES (5). Los fenómenos vasculíticos en el parénquima pulmonar pueden producir HTP crónica y progresiva que comparte patogénesis y tratamiento con la HTP primaria. En segundo lugar, existe mayor predisposición a desarrollar tromboembolismo pulmonar, sobre todo si se asocia la presencia de anticoagulante lúpico. Por último, se ha descrito recientemente el denominado síndrome de hipoxemia reversible aguda $(6,7)$, que parece deberse a la adhesión y acúmulo de neutrófilos en los pequeños vasos pulmonares secundarios a la activación endotelial y del complemento (8). Dada la aparición y desaparición rápida de la HTP y la ausencia de datos sugerentes de embolismo pulmonar en nuestra paciente, es poco probable que tuviese relación con las dos primeras entidades mencionadas.
Como ilustra el presente caso, aunque el LES es una causa rara de taponamiento cardiaco $(9,10)$, conviene considerarla dentro del diagnóstico etiológico, independientemente de la edad del paciente.

\section{J. Polo Sabau, J. L. Gutiérrez Chico', O. Sabillón², M. Díaz Curiel}

Servicios de Medicina Interna, ${ }^{1}$ Cardiología y ${ }^{2}$ Neumología. Fundación Jiménez Díaz. Madrid

1. Zashin SJ, Lipsky PE. Pericardial tamponade complicating systemic lupus erythematosus. J Rheumatol 1989; 16: 374-377.

2. Reiner JS, Furie RA. Cardiac tamponade as an initial manifestation of systemic lupus erythematosus. J Rheumatol 1989; 16: 1127-1129.

3. De Inocencio J, Lovell DJ. Cardiac function in systemic lupus erythematosus. J Rheumatol 1994; 21: 2147-2156.

4. Moder KG, Miller TD, Tazelaar HD. Cardiac involvement in systemic lupus erythematosus. Mayo Clin Proc 1999; 74: 275-284.

5. Keane MP, Lynch JP. Pleuropulmonary manifestations of systemic lupus erythematosus. Thorax 2000; 55: 159-166.

6. Abramson SB, Dobro J, Eberle MA, et al. Acute reversible hypoxemia in systemic lupus erythematosus. Ann Intern Med 1991; 114: 941-947.

7. Martínez-Taboada VM, Blanco R, Armona J, et al. Acute reversible hypoxemia in systemic lupus erythematosus: a new syndrome or an index of disease activity?. Lupus 1995; 4: 259-262.

8. Belmont HM, Buyon J, Giorno R, et al. Up-regulation of endothelial cell adhesion molecules characterizes disease activity in systemic lupus erythematosus. The Schwartzman phenomenon revisited. Arthritis Rheum 1994; 37: 376-383

9. Lorell B. Pericardial diseases. En: Braunwald E editor. Heart Disease. $5^{\text {a }}$ ed. Filadelfia: Saunders, 1997; 1478-1534.

10. Aróstegui J, García ML, Galíndez E, et al. Taponamiento cardiacco como manifestación inicial del lupus eritematoso sistémico. An Med Interna (Madrid) 2001; 18: 451-452.

Pseudotumor inflamatorio de bazo: presentación de un caso con clínica trombopénica y revisión de la literatura

\section{Sr. Director:}

El pseudotumor inflamatorio es una lesión poco frecuente, de probable origen reparativo (1). Ha sido descrito en múltiples localizaciones, siendo las más frecuentes pulmón, tracto gastrointestinal e hígado $(1,2)$. En el bazo es una lesión muy rara. La lesión no se presenta con una clínica definida, por lo que muchas veces el diagnóstico es casual y en un menor número de casos se realiza en el estudio de otras patologías como anemias o púrpura trombocitopénica idiopática (PTI) (3). Presentamos un nuevo caso de pseudotumor inflamatorio de bazo en una paciente en estudio por PTI y gammapatía policlonal, cuya exploración radiológica mostró una tumoración esplénica.

Paciente de 60 años que acude a urgencias hace cinco años por presentar hematomas y petequias. Como antecedentes personales refiere histerectomía 13 años antes por causas desconocidas y un accidente de tráfico hace un año con traumatismo en hipocondrio izquierdo. La exploración analítica mostró 10.000 plaquetas, siendo los factores de coagulación, hemograma, VSG, bioquími- 
ca, proteinograma e inmunoelectroforesis normales. Marcadores de hepatitis B y C, anticuerpos antinucleares y factor reumatoide negativos. Ecografía abdominal normal. Estudio de médula ósea (punción y biopsia) normal. Se instauró un tratamiento esteroideo con respuesta irregular, produciéndose remisiones y recidivas. A partir del primer año, se detectaron alteraciones analíticas: VSG 70, ASLO 360, FFAA 380, PC reactiva 6 y Gammapatía policlonal con aumento de IgA e IgG. Las exploraciones radiológicas, ecografía y TAC abdominal evidenciaron una masa esplénica de $5 \mathrm{~cm}$. bien delimitada, sin otros hallazgos. Serie ósea normal. La trombopenia y los parámetros analíticos se normalizaron con la reinstauración de la terapia esteroidea. Tras el cese del tratamiento se originaron nuevas recaídas, llegando a alcanzar las ASLO valores de 925 y acompañándose de dolores óseos. Un año después en las determinaciones analíticas se detectaron Ac antimúsculo liso, antireticulina y antigliadina. La TSH-T4 y beta2 microglobulina en suero fueron normales. Proteína de Bence Jones en orina negativa. La endoscopia gastroduodenal y biopsia resultaron inespecifícas. La exploración radiológica no mostró variaciones de la lesión esplénica. Ante nuevas recidivas de la trombopenia se decidió la esplenectomía diagnóstica y terapéutica que se realizó sin incidencias. De inmediato se normalizaron las cifras de plaquetas. Un año después la paciente se encuentra asintomática y los parámetros analíticos son todos normales.

En los cortes seriados del bazo se apreció un nódulo redondeado, grisáceo y homogéneo, bien delimitado, no encapsulado, de 5 x $5.5 \mathrm{~cm}$. (Fig. 1). El resto del parénquima esplénico presentaba un aspecto normal. El estudio histológico mostró un estroma con componente fibroblástico y producción de fibras de reticulina y colágena y otro inflamatorio constituído por linfocitos y células plasmáticas. En todos los casos destacaba la ausencia de atipicidad y actividad proliferativa en las células. No se observaron células de tipo Reed-Sternberg.

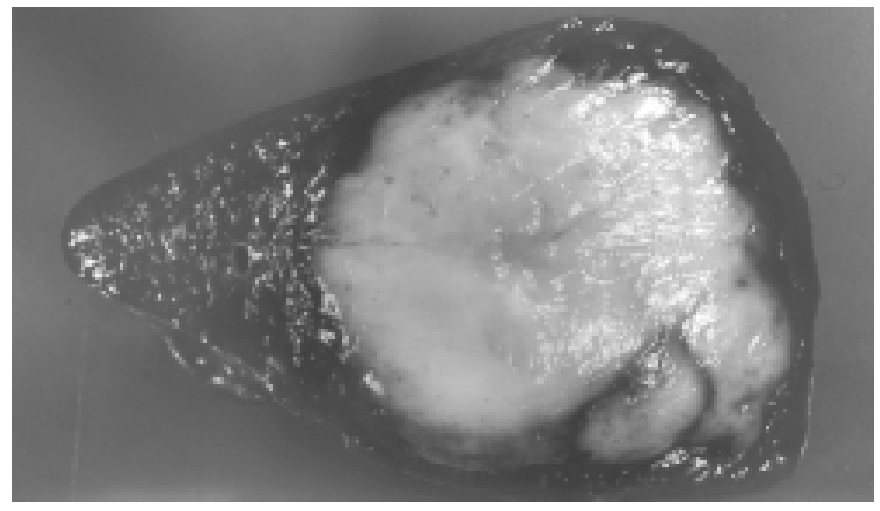

Fig. 1.- Imagen macroscópica de una sección del bazo. O bsérvese el aspecto de malignidad de la lesión.

El resultado del estudio inmunohistoquímico fue el siguiente: negativa la expresión de queratina, desmina, PS100 y CD30. La vimentina fue +++, con expresión tanto en el componente estromal como en las paredes vasculares. La actina, +++ en el músculo liso de los vasos sanguíneos y + focalmente en las células del estroma. El PAN-B +++ en aisladas células linfoides mientras que el PAN-T +++ se apreció en la mayoría de aquellas. Se observó expresión de Kappa +++ y Lambda +++ por igual en la mayor parte del componente linfoplasmocitoide lo que permitió establecer la policlonalidad de dichas células. La expresión de CD68 y lisozima fue focal y débil (+).

El pseudotumor inflamatorio es un tumor poco frecuente que ha sido descrito hasta la fecha en prácticamente todos los siste- mas y la mayoría de sus órganos $(1,3)$. En el bazo se han descrito 35 casos (2-7) desde 1984 en que se publicó por primera vez Cotelingam y Jaffe (1). El interés del caso no se refiere tan sólo a su rareza, sino al diagnóstico diferencial que plantea con procesos tanto benignos como malignos (7).

Se manifiesta preferentemente en la quinta y sexta décadas, sin preponderancia por sexo alguno $(3,6)$. En más de un $50 \%$ de los casos se presenta como hallazgo casual en autopsias o en exploraciones por otras patologías $(5,6)$ tal como ocurrió en nuestro caso, sin embargo, la manifestación inicial con síntomas de PTI es rara y similar a la descrita por Thomas en tres de sus casos (6). La forma más frecuente de presentación es dolor en hipocondrio izquierdo que en ocasiones se acompaña de fiebre o esplenomegalia $(3,6)$.

El diagnóstico casi nunca es preoperatorio. De hecho, los hallazgos radiológicos son bastante inespecíficos $(2,3)$. El diagnóstico diferencial clínico-radiológico se plantea frente a una masa esplénica, generalmente única y bien definida, con procesos benignos como hamartoma, absceso, infarto, lesión inflamatoria $\mathrm{u}$ otros tumores y malignos tales como linfoma, otras neoplasias o metástasis (2-4).

Macroscópicamente suelen ser masas únicas, bien delimitadas, blancogrisáceas y homogéneas $(5,6)$, similar al aspecto de nuestra lesión. Sin embargo en ocasiones pueden tener zonas quísticas o áreas blancas de aspecto necrótico (6). El aspecto macroscópico de malignidad observado en nuestro caso, ha sido también constatado en otros trabajos (3).

Comentamos en este trabajo el valor diagnóstico del estudio anatomopatológico e inmunohistoquímico especialmente en el diagnóstico diferencial con otras entidades que se presentan en el bazo como nódulos solitarios, tales como linfoma maligno (Hodgkin y no Hodgkin), pseudolinfoma, fibrohistiocitoma maligno, plasmocitoma $(2,5)$ tumores metastásicos y procesos benignos como hamartoma esplénico, lesiones vasculares, infecciones granulomatosas, quistes e hiperplasia linfoide localizada. La característica distribución zonal que puede apreciarse también radiológicamente y el inmunofenotipo policlonal, ayudan a establecer un correcto diagnóstico. En nuestro caso se planteó el diagnóstico diferencial con el fibrohistiocitoma por la presencia de zonas fusocelulares con patrón verticilado, pero la ausencia de atípias y actividad proliferativa declinó el diagnóstico. Además la positividad para marcadores histiocitarios era débil, focal e irregular. El diagnóstico de linfoma, tanto Hodgkin como no Hogdkin, se eliminó por la ausencia de células de Reed. En las primeras descripciones del pseudotumor inflamatorio en bazo se planteó el diagnóstico diferencial con el pseudolinfoma (4), sin embargo este último suele poseer un predominio linfoide, generalmente $\mathrm{B}$, además de hiperplasia folicular, mientras que el pseudotumor inflamatorio contiene más estroma e infiltrado inflamatorio mixto (1). Algún autor pone en duda la existencia de pseudolinfomas en bazo, aduciendo que los casos descritos probablemente sean linfomas bien diferenciados de patrón folicular (4). En nuestro caso predominaron en las células linfoides los marcadores positivos $\mathrm{T}$ frente a los B, observación realizada también por otros autores $(3,6)$.

La causa del pseudotumor inflamatorio de bazo es desconocida. Existen tres teorías que intentan explicar el origen (3). La hipótesis menos probable es la infecciosa, dado que en pocos casos se describen datos que puedan avalar esta teoría (4). En nuestro caso no se realizaron cultivos, pero los hallazgos clínicos y morfológicos no inducían a pensar en una etiología infecciosa. La teoría vascular, propone una obstrucción vascular o una hemorragia intraparenquimatosa relacionada con un traumatismo o una coagulopatía de consumo $(3,6,7)$. Por último, el origen autoinmune lo apoyan la coexistencia de PTI en varios de los casos descritos (3,5-7). En nuestro caso coexisten la PTI y el antecedente de un traumatismo en hipocondrio izquierdo. En cualquier caso, tanto si se trata de un origen vascular como si 
coexiste una PTI, la hemorragia intraparenquimatosa parece ser el origen más probable de la lesión (3).

\section{R. J. Bertolín Bernades, J. Ferrando-Marco', $\mathbf{M}^{\mathrm{a}}$ J. Arilla- Morell $^{2}$, A. Pallas-Regueira}

Servicios de Cirugía General, ${ }^{1}$ Anatomía Patológica, ${ }^{2}$ Hematología. Hospital de Sagunto. Valencia

1. Warnke RA, Weiss LM, Chan JK, Cleary ML, Dorfman RF. Inflammatory pseudotumor of spleen, in rare lesions of the spleen. Tumors of the lymph nodes and spleen. Atlas of Tumor Pathology A.F.I.P. Bethesda, 1995. p. 502-503.

2. Hashizume TK, Kawanaka MS, Sugimachi K, Maeda IH, Akaboshi K. Laparoscopic splenectomy for an inflammatory pseudotumor of the spleen. Operative technique and case report. Hepatogastroenterology 1998; 45 (23): 1887-1991.

3. Galindo-Gallego M, Ortega-Serrano MP, Ortega-López M, EstebanCollazo F, Guinea-Esquerdo L. Inflammatory pseudotumor of the spleen. Report of two cases and literature rewiew. Minerva Chir 1997; 52: 13791388.

4. Wiernik PH, Rader M, Becker NH, Morris SF. Inflammatory pseudotumor of spleen. Cancer 1990; 66: 597-600.

5. Monforte-Muñoz H, Ro JY, Manning JT, Landom G, Del Junco G, Carlson TS, Ayala AG. Inflammatory Pseudotumor of the Spleen. Report of two cases with a Review of the literature. Am J Clin Pathol 1991; 96: 491-495.

6. Thomas RM, Jaffe ES, Zarate-Osorno A, Jeffrey-Medeiros L. Inflammatory Pseudotumor of the Spleen. A Clinicopathologic and Immunophenotypic Study of eight cases. Arch Pathol Lab Med 1993; 117: 921926.

7. Dalal BI, Greeenberg H, Quinonez GE, Gough JC. Inflammatory Pseudotumor of the Spleen. Morphological, Radiological, Immunophenootypic and ulttrastructural features. Arch Pathol Lab Med 1991; 115: 1062 1064

\section{Enfermedad de Hodgkin epitroclear. Aportación de un nuevo caso}

\section{Sr. Director:}

Hemos leído con interés el artículo publicado por Jiménez y colaboradores en el que se describe un paciente con Enfermedad de Hodgkin de comienzo epitroclear (1). Aportamos un nuevo caso con presentación y recaída a ese nivel.

Varón de 29 años de edad, sin antecedentes patológicos de interés que consultó por tumoración en codo derecho de $2 \mathrm{~cm}$ de diámetro mayor, de 9 meses de evolución y aparición tras traumatismo. Negaba cualquier sintomatología acompañante. Se procedió a exéresis de la lesión con resultado histológico de linfoma de Hodgkin tipo predominio linfocítico. Se nos envió para valoración de tratamiento complementario. El estudio de extensión (exploración física, TAC toracoabdominal, biopsia de médula ósea y PET) fue negativo. Pendiente de los resultados del estudio realizado notó la reaparición de un nuevo nódulo en la misma localización. Se procedió a una nueva exéresis de la lesión con resultado de linfoma de Hodgkin. Iniciamos tratamiento con esquema ABVD cada 15 días (adriamicina $25 \mathrm{mg} / \mathrm{m}^{2}$, bleomicina $10 \mathrm{mg} / \mathrm{m}^{2}$, vinblastina $6 \mathrm{mg} / \mathrm{m}^{2}$ y dacarbacina $375 \mathrm{mg} / \mathrm{m}^{2}$ ) durante 3 ciclos y radioterapia, 30 Gy sobre el lecho de resección de los nódulos. El paciente permanece asintomático y sin evidencia de recaída 4 meses después de finalizado el tratamiento.

Habitualmente las adenopatías epitrocleares son de origen infeccioso o bien aparecen en el contexto de enfermedades como la sarcoidosis . La etiología linfomatosa es especialmente infrecuente, más aún la enfermedad de Hodgkin (1-3) y habitualmente aparece en el contexto de un linfoma no Hodgkin en estadios avanzados. En nuestro Servicio, con amplia experiencia en el tratamiento de linfomas es el primer caso de enfermedad de Hodgkin con afectación epitroclear como forma de presentación.

\section{Vicente Baz, M. Ruiz Borrego, E. Arriola Arellano, A. Fer- nández-Freire, J. A. Moreno Nogueira}

Servicio de Oncología Médica. Hospitales Universitarios Virgen del Rocío. Sevilla.

1. Jiménez Gonzalo FJ, Medina Pérez M, Ríos Herranz E. Enfermedad de Hosgkin de comienzo epitroclear. An Med Interna 2001; 18: 554.

2. Ghirardelli ML, Jemos V, Gobbi P. Diagnostic approach to lymph node enlargament. Haematologica 1998; 84: 242-7.

3. Yu A, Steinfeld AD. Hodgkin's disease presenting in epitrochlear nodes. Med Pediatr Oncol 1984; 12: 244-6.

\section{Pseudomigraña con pleocitosis. Un nuevo caso}

\section{Sr. Director:}

En 1981 Bartleson y cols. (1), utilizan por primera vez el término pseudomigraña con pleocitosis (PMP) para definir un proceso benigno y autolimitado, que se presenta con un déficit neurológico transitorio, cefalea de tipo migrañoso y pleocitosis en el líquido cefalorraquídeo (LCR). Desde entonces sólo se han descrito alrededor de cien casos en la literatura médica (2). Dado que es importante reconocer este síndrome y diferenciarlo de otras urgencias neurológicas más graves para evitar exploraciones agresivas, creemos de interés la comunicación del siguiente caso.

Se trata de un varón de 21 años sin antecedentes de interés que acudió al servicio de urgencias tras despertarse de madrugada con cefalea intensa hemicraneal izquierda, acompañada de parestesias en extremidades derechas, náuseas, vómitos y afasia motora, en los 15 días previos refería dos episodios similares autolimitados. La exploración física mostraba una temperatura de 39 grados y ligera disminución del surco nasogeniano derecho. La analítica era normal, salvo una glucemia de $143 \mathrm{mg} / \mathrm{dl}$, con 10.840 leucocitos $/ \mathrm{mm}^{3}$ (87\% neutrófilos y $9,4 \%$ linfocitos) y una VSG de $6 \mathrm{~mm}$ en la primera hora. El electrocardiograma y la radiografía de tórax fueron normales, así como el TAC y la RMN cerebral. Una primera punción lumbar mostró un líquido claro y transparente, con 65 células blancas ( $90 \%$ mononucleadas) y 70 hematíes por $\mathrm{mm}^{3}$; proteínas $129,9 \mathrm{mg} / \mathrm{dl}$, glucosa $78 \mathrm{mg} / \mathrm{dl}$. El cuadro cedió completamente en diez horas y posteriormente permaneció asintomático y afebril. El día del episodio se realizó un electroencefalograma (EEG) que presentaba trenes bilaterales de actividad lenta con predominio en hemisferio izquierdo, de persistencia moderadaalta, sin actividad asociada de forma específica a epilepsia. Al cuarto día el EEG era ya normal. Al tercer día se obtuvo un nuevo LCR transparente con 155 células blancas $/ \mathrm{mm}^{3}$ (95\% mononucleares), proteínas $158 \mathrm{mg} / \mathrm{dl}$ y glucosa $54 \mathrm{mg} / \mathrm{dl}$ con estudio citológico benigno y discreta reacción histiocitaria. La electroforesis del LCR mostró un aumento policlonal de $\operatorname{IgG}(\operatorname{IgG}=9 \mathrm{mg} / \mathrm{dl})$. Resultaron normales o negativas las siguientes determinaciones: hemocultivos, serología en sangre de enterovirus ECHO-coxsackie, virus herpes simple 1 y 2, parotiditis, sarampión, HIV y Borrelia burdogferi, serología en LCR de enterovirus ECHO-cox- 
sackie, virus herpes simple 1 y 2 , parotiditis, sarampión y varicela-zoster. Posteriormente el paciente permaneció asintomático durante un año de seguimiento.

La PMP es más frecuente en varones en la tercera y cuarta década de la vida. En aproximadamente la mitad de los pacientes se encuentran síntomas de enfermedad viral o fiebre. En una serie de 50 casos con PMP (3), las manifestaciones neurológicas más frecuentes fueron sensitivas $(70 \%)$, seguidas de la afasia $(66 \%)$, las alteraciones motoras $(42 \%)$ y, por último, los síntomas visuales $(18 \%)$. De la combinación de síntomas, la afasia motora con déficit sensitivo y motor en el hemicuerpo derecho fue la más común. La duración de los síntomas neurológicos va de 5 horas a 3 días. La cefalea es moderada-severa, pulsátil en la mayoría de los casos, más frecuentemente bilateral, de duración variable entre una hora y una semana. Se puede acompañar de náuseas y/o vómitos, fotofobia y sonofobia. Nunca se encuentran signos meníngeos (4). Los pacientes tienen de 1 a 11 episodios a lo largo de 12 semanas, permaneciendo asintomáticos con un seguimiento

\begin{tabular}{|c|c|c|}
\hline \multicolumn{3}{|c|}{ TABLA I } \\
\hline & M igraña con aura & PM P \\
\hline Sexo & $\begin{array}{l}\text { Más frecuente en } \\
\text { mujeres }\end{array}$ & $\begin{array}{l}\text { Más frecuente en } \\
\text { varones }\end{array}$ \\
\hline $\begin{array}{l}\text { Historia } \\
\text { personal de } \\
\text { migraña }\end{array}$ & M uy frecuente & Ausente \\
\hline $\begin{array}{l}\text { Historia } \\
\text { familiar de } \\
\text { migraña }\end{array}$ & Frecuente & Ausente \\
\hline $\begin{array}{l}\text { Historia } \\
\text { natural }\end{array}$ & Recidivante & Benigna \\
\hline $\begin{array}{l}\text { Signos focales } \\
\text { transitorios }\end{array}$ & O casionalmente & M uy frecuentes \\
\hline $\begin{array}{l}\text { Duración } \\
\text { déficit } \\
\text { neurológico }\end{array}$ & $\begin{array}{l}\text { El aura dura menos de } \\
\text { una hora y los síntomas } \\
\text { visuales son } \\
\text { prácticamente } \\
\text { constantes }\end{array}$ & $\begin{array}{l}\text { Duración media de } \\
\text { cinco horas }\end{array}$ \\
\hline $\begin{array}{l}\text { Pleocitosis } \\
\text { LCR }\end{array}$ & $\begin{array}{l}\text { Rara. Debe reconocerse } \\
\text { la posibilidad de } \\
\text { ataques de migraña } \\
\text { con pleocitosis en LCR, } \\
\text { sin embargo, tal } \\
\text { combinación es } \\
\text { extremadamente rara. } \\
\text { Ausencia de aumento } \\
\text { de proteínas totales o } \\
\text { aumento de presión en } \\
\text { LCR }\end{array}$ & $\begin{array}{l}\text { Siempre. Proteínas } \\
\text { aumetadas y elevada } \\
\text { a la presión de } \\
\text { apertura }\end{array}$ \\
\hline $\begin{array}{l}\text { Alteración } \\
\text { EEG }\end{array}$ & O casionalmente & Frecuente \\
\hline Tratamiento & Difícil & No precisa \\
\hline Evolución & Crónica & $\begin{array}{c}\text { Curación espontánea, } \\
\text { no cefaleas crónicas }\end{array}$ \\
\hline Localización & Hemicraneal & Suele ser bilateral \\
\hline
\end{tabular}

de entre 6 meses y 10 años. En 1995 Berg y cols., propusieron unos criterios diagnósticos de esta entidad (5). Las extensas determinaciones serológicas, microbiológicas y inmunológicas son normales o negativas. Un hecho distintivo de PMP es la pleocitosis linfocitaria en el LCR durante el periodo sintomático, así como punción final normal. La mayoría tienen elevación de la presión de apertura y aproximadamente el $90 \%$ tienen aumentadas las proteínas totales en la primera punción lumbar, la glucosa suele ser normal. Aproximadamente el $72 \%$ de los pacientes con pseudomigraña presentan en el EEG ondas lentas focales en el lado de origen de los síntomas neurológicos, durante los días de la crisis, y a veces incluso en las intercrisis (4) normalizándose tras el periodo sintomático. En el diagnóstico diferencial se incluyen muchas condiciones: esclerosis múltiple, enfermedad de Lyme, neurosífilis, neurobrucelosis, infección por micoplasma, meningitis por VIH, meningitis granulomatosa, aracnoiditis neoplásica, meningitis de Mollaret y la angiografía convencional que puede causar pleocitosis polimorfonuclear y déficit neurológicos; sin embargo, la migraña es el primer diagnóstico que se plantea, aunque las alteraciones del LCR hacen dudar sobre este diagnóstico. La cefalea de PMP tiene características de migraña con aura, pero existen características diferenciadoras entre ambas (Tabla I). La etiopatogenia y fisiopatología de este síndrome son desconocidas. Los primeros casos, desataron la polémica entre los que sostienen que el cuadro sería explicable por una reacción meníngea condicionada por los episodios de migraña con aura, y los que defienden que lo primario sería al afectación meningo-encefalítica que condicionaría secundariamente una cefalea de características migrañosas. En 1995, Serrano y cols. (6) sugieren una tercera hipótesis "inmunogénica ", que sugería que hasta un tercio de los pacientes tenían antecedentes de fenómenos capaces de estimular el sistema inmunitario (casi siempre un cuadro vírico) en las dos semanas previas, proponiendo que la migraña con pleocitosis sería secundaria a una inflamación aséptica de los vasos leptomeningeos condicionado por una activación del brazo humeral del sistema inmune. Los estudios recientes de la hemodinámica cerebro-vascular con eco-doppler transcraneal y SPECT (7-10), sugieren que la explicación fisiopatológica de los fenómenos deficitarios, también debería ser similar a la de la migraña con aura, esto es un fenómeno de depresión cortical prolongada, identificando la PMP como un auténtico síndrome migrañoso, inmunomediado, habitualmente post-infeccioso y de evolución autolimitada.

\section{B. de Rivas Otero, P. Rivas González}

Servicio de Neurología. Fundación Jiménez Díaz. Madrid

1. Bartleson JD, Swanson JW, Whisnant JP. A migrainous syndrome with cerebrospinal fluid pleocytosis. Neurology 1981; 31: 1257-62.

2. Jover-Sáenz A, Porcel-Pérez JM, Rubio-Caballero M. Pseudomigraña con síntomas neurológicos transitorios y pleocitosis del líquido cefalorraquídeo. Rev Neurol 1999, 28: 436-438.

3. Gómez-Aranda F, Canadillas F, Martí-Massó JF, Díez-Tejedor E, Serrano PJ, Leira R, et al. Pseudomigraine with temporary neurological symptoms and lymphocytic pleocytois. A report of 50 cases. Brain 1997; 120: 1105-1113.

4. Epelde Gonzalo F, Bonaventura Ibars I, Tomás Vecina S. Migraña con pleocitosis, una entidad poco conocida. An Med Interna (Madrid) 1996; 13: $152-153$

5. Berg MJ, Williams LS. The transient syndrome of headache with neurologic deficits and CSF lymphocytosis. Neurology 1995; 45: 1648-54.

6. Serrano PJ, Arnal C, Carnero C, Mínguez A, Foronda J, Hernández FJ. Síndrome de migraña con pleocitosis de LCR. Aportación de 4 nuevos casos y revisión de la literatura. Rev Neurol 1994; 23: 756-759.

7. Serrano-Castro PJ, Amrani Y, Olivares-Romero J. Hemodinámica cerebral en el síndrome de pseudomigraña con pleocitosis de LCR: un estudio doppler transcraneal. Rev Neurol 2000; Sup1. 15; 31: 407-411. 
8. Caminero AB, Pareja JA, Arpa J, Vivancos F, Palomo F, Coya J. Migrainous syndrome with CSF pleocytosis. SPECT findings. Headache 1997; 37: 511-515.

9. Fuentes B, Díez Tejedor E, Frank A. Syndrome of headache with neurological deficits and CSF lymphocytosis: A spreading depression mechanism? Headache 1998; 38: 324

10. Fuentes B, Díez Tejedor E, Pascual J, Coya J, Quirce R. Cerebral blood flow changes in pseudomigraine with pleocytosis analyzed by single photon emission computed tomography. A spreading depression mechanism? Cephalalgia 1998; 18: 570-3.

\section{Endocarditis aislada de válvula pulmonar en un corazón sano}

\section{Sr. Director:}

La "endocarditis infecciosa aislada de válvula pulmonar" (EIP) es muy rara (0-1,5\% del total), siendo excepcional en ausencia de factores predisponentes $(1,2)$. Presentamos un caso de EIP en una paciente sana con el fin de aportar nuestra experiencia y orientación terapéutica.

Mujer de 68 años de edad, sin antecedentes personales ni epidemiológicos de interés, ingresada por escalofríos, fiebre de unos 6 días de evolución y discreta desorientación en las horas previas. Se había quemado la pierna izquierda con agua caliente unos días antes. En la exploración física presentaba TA 130/65 $\mathrm{mmHg}, \mathrm{T}^{\mathrm{a}}$ $37^{\circ} \mathrm{C}$, sequedad mucocutánea, desorientación, bradipsiquia, crepitantes finos bibasales, soplo diastólico en borde esternal izquierdo III/VI y una quemadura de segundo grado en la rodilla izquierda sin signos de infección, siendo normal el resto. En la analítica destacaba: 16.700/ml leucocitos con 92\% neutrófilos; $\mathrm{Hg} 11 \mathrm{~g} / \mathrm{dl}$, Hcto 32,9\%, VCM $81.2 \mathrm{fl}$, plaquetas 42.000/ul; VSG $110 \mathrm{~mm} / \mathrm{h}$; glucosa $121 \mathrm{mg} / \mathrm{dl}$, urea $106 \mathrm{mg} / \mathrm{dl}$, LDH $559 \mathrm{UI} / \mathrm{L}$, GOT $61 \mathrm{UI} / \mathrm{L}$, GPT $37 \mathrm{UI} / \mathrm{L}, \mathrm{FA} 547 \mathrm{UI} / \mathrm{L}$, GGT $120 \mathrm{UI} / \mathrm{L}$ y albúmina $2 \mathrm{~g} / \mathrm{dl}$; creatinina e iones normales. En la coagulación: actividad de TP $77 \%$, TTPA 42,3 seg, fibrinógeno $675 \mathrm{mg} / \mathrm{dl}$, $\mathrm{PDF}+20$. La GAB: pH 7,41, pO2 70, S02 94,4\%, pCO2 32, $\mathrm{C} 03 \mathrm{H}$ 20. La radiografía de tórax y el ECG iniciales eran normales.

A las $24 \mathrm{~h}$ del ingreso se informó del crecimiento de cocos grampositivos en los hemocultivos obtenidos en urgencias, iniciándose tratamiento con vancomicina $2 \mathrm{~g}$ /día más gentamicina $240 \mathrm{mg} / \mathrm{día}$, y se identificaron después como Staphylococcus aureus meticilinsensible. El ecocardiograma transesofágico confirmó el hallazgo de una lesión vegetativa de $5 \mathrm{~cm}$ en la válvula pulmonar, sin otras anomalías (Fig. 1).

Una semana después presentó hemoptisis con edema intersticial pulmonar y derrame pleural izquierdo en la radiología simple, así como nódulos cavitados diseminados e infarto séptico basal izquierdo en el TAC torácico. Posteriormente se deterioró la función renal y se envió a cirugía cardiaca. Fué intervenida en $72 \mathrm{~h}$ colocándose un homoinjerto pulmonar criopreservado con buena evolución a los 12 meses de seguimiento, permaneciendo asintomática y sin datos de insuficiencia actual.

El primer caso de EIP fue publicado en 1964 por Levin, desde entonces sólo se han descrito algunos casos, incluyendo éste, en personas sanas y sin antecedentes patológicos (1)

En los países desarrollados la causa más frecuente de endocarditis derecha es el abuso de drogas intravenosas $(0,75-3,5 \%)$; también se han asociado a cardiopatías y broncopatías congénitas intervenidas o no, valvuloplastia pulmonar, hemodiálisis, cateterismos venosos centrales, cables de marcapasos e infecciones sistémicas $(2,3)$. Se ha visto un incremento en ancianos, poblaciones

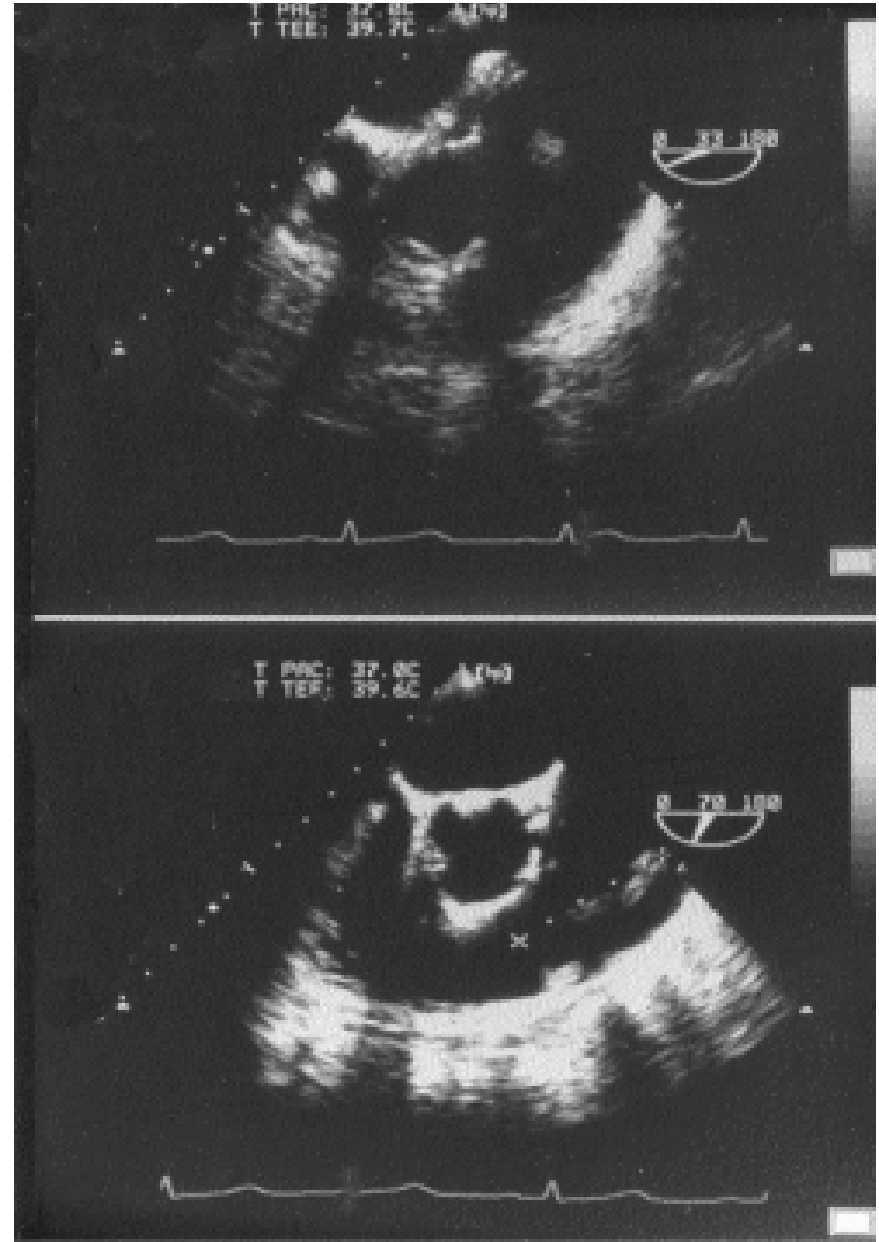

Fig. 1. Ecocardio transesofágico: dilatación aneurismática del tronco de la pulmonar con lesión vegetativa de unos $5 \mathrm{~cm}$.

de riesgo y un aumento del porcentaje de casos por Stafilococcus aureus, todo ello en probable relación con una mayor manipulación $(4,5)$. La búsqueda de puertas de entrada, prevención, detección precoz y un tratamiento inmediato y agresivo van a suponer un reto en los próximos años $(6,7)$.

La experiencia con respecto a su manejo aún está basada en casos individuales y, por tanto, tienen un pronóstico variable $(6,7,8)$. Las indicaciones quirúrgicas son conocidas: fiebre persistente pese a antibioterapia correcta, insuficiencia cardíaca, algunos tipos de gérmenes (Serratia sp, Pseudomona sp, hongos), deterioro hemodinámico, émbolos sépticos y vegetaciones mayores de $1 \mathrm{~cm}$.

Entre las posibilidades quirúrgicas, el homoinjerto criopreservado tiene menos riesgo de degeneración valvular y menos complicaciones con mayor supervivencia. En la actualidad esta técnica está considerada el tratamiento de elección para reconstruir el tracto de salida del ventrículo derecho. No precisa anticoagulación y se considera ideal en niños, jóvenes y adultos con vida basal activa $(9,10)$.

A. M ${ }^{a}$ Bravo Blanco, F. de la Peña ${ }^{1}$, M. García ${ }^{1}$, F. Redondo ${ }^{1}$, R. Barreiro, O. Fernández, G. Pradas ${ }^{2}$

Servicio de Medicina Interna. Departamento de Infecciosas. ${ }^{1}$ Servicio de Cardiología. Complexo Hospitalario de Ourense. Ourense. ${ }^{2}$ Servicio de Cirugía Cardiaca. MEDTEC-Vigo 
1. Hussain KMA, Kabins S, Lieb D, Chandna H and Denes P. Coagulasenegative Staphylococcus endocarditis restricted to the normal pulmonic valve in a patient with end-stage renal disease: a case report and review. CID 1998; 27: 1550-2.

2. De Alarcón A, Villanueva JL. Endocarditis en adictos a drogas por vía parenteral. Endocarditis derecha. Influencia de la situación VIH. Rev Esp Cardiol. 1998; 51 (Supl. 2): 71-78.

3. Ramadan FB, Beanlands DS, et al. Isolated pulmonic valve endocarditis in healthy hearts: a case report and review of the literature. Can J Cardiol 2000;16 (10):1282-8.

4. Rivera Casado JM. Endocarditis infecciosa en el anciano. Rev Esp Cardiol 1998; 5 (Supl. 2): 64-70.

5. Pérez Paredes M, Ramos Rincón JM, Carnero Varo A, et al. Endocarditis pulmonar aislada sobre válvula nativa en un paciente anciano sin factores predisponentes. Rev Esp Cardiol 2001; 54 (3): 402-404.

6. Schaefer A, Meyer GP, Waldow A, Weiss T, Hausmann D, Drexler H. Pulmonary valve endocarditis. Circulation 2001; 6; 103 (9): E53-4.

7. Mantia M, Lefebvre $P$, Friart A. Isolated pulmonary valve endocarditis: a case report. Arch Mal Coeur Vaiss 2001; 94 (3): 223-5.

8. Lagarto V, Cabral S, Oliveira F, Pereira S, et al. Isolated infective endocarditis of pulmonary valve in patient with interventricular septal defect. Rev Port Cardiol 2000; 19 (5): 617-21.

9. De la Fuente A, Sánchez R, Fernández J, Romero J, et al. Sustitución valvular pulmonar por endocarditis. A propósito de un caso. Cir Cardiov 2000; 7; 2: 85-86.

10. Llosa JC, Gosalbez F, Cofiño JL, Naya JL, Valle JM. Pulmonary valve endocarditis: mid-term follow up of pulmonary valvectomies. J Heart Valve Dis 2000; 9; 3 (5): 359-363.

\section{Afectación cutánea y síndrome de vena cava superior: presentación insólita de la enfermedad de Hodgkin}

\section{Sr. Director:}

La enfermedad de Hodgkin (EH) es una neoplasia hematológica cuya presentación clínica y afectación tisular pueden ser muy variables. La forma de presentación más frecuente es la aparición de adenopatías periféricas. El diagnóstico de $\mathrm{EH}$ a partir de un síndrome de vena cava superior (SVCS) es excepcional. La afectación cutánea asociada a la EH incide en un $17-53 \%$ (1). La mayoría de los enfermos presentan lesiones inespecíficas existiendo un escaso porcentaje de manifestaciones especificas y de forma de EH exclusivamente cutánea. Todas estas lesiones resultan insólitas antes de esta- blecer el diagnóstico de EH. Presentamos el caso de una mujer con cuadro cutáneo previo que debutó con SVCS.

Mujer de 53 años de edad que ingresó por edema en cara y miembros superiores. Entre sus antecedentes destacaban ser portadora heterocigota del factor $\mathrm{V}$ Leiden, cólicos nefríticos de repetición, distimia y un cuadro dermatológico abigarrado de dos años de evolución. Las lesiones cutáneas tenían una apariencia urticariforme, se localizaban en áreas extensoras de extremidades y en ocasiones se tornaban vesiculosas. Cursaban en brotes, sin un desencadenante claro. Las repetidas biopsias mostraron reacción urticarial en unas ocasiones y dermatitis herpetiforme con inmunofluorescencia directa no concluyente en otras. Estos episodios de afectación cutánea mejoraban con antihistamínicos y ciclos cortos de corticoides orales. Tras año y medio de evolución la paciente ingresó por anemia normocítica normocrómica acompañada de un nuevo exantema y fue diagnosticada histológicamente de vasculitis leucocitoclástica (VL).

En la exploración física destacaba edema de cara y extremidades superiores con ingurgitación yugular y masa palpable en fosa supraclavicular derecha. Analítica: hematíes 4.230.000, hemoglobina 10,7 g/dL, hematocrito 33,4\%, VCM 76,2, HCM 25,2, leucocitos 18.150 (neutrófilos 14.770, linfocitos 1910, monocitos 740), plaquetas 372.000, VSG $84 \mathrm{~mm}$. GOT-AST $15 \mathrm{U} / \mathrm{L}$, GPTALT $10 \mathrm{U} / \mathrm{L}$, GGT $10 \mathrm{U} / \mathrm{L}, \mathrm{LDH} 271 \mathrm{U} / \mathrm{L}$, urea $59 \mathrm{mg} / \mathrm{dL}$, creatinina $1,15 \mathrm{mg} / \mathrm{dL}$, glucosa $86 \mathrm{mg} / \mathrm{dL}, \beta_{2}$ microglobulina 4.4 $\mathrm{mg} / \mathrm{dL}$, sideremia $14 \mathrm{mg} / \mathrm{dL}$, ferritina $101 \mathrm{ng} / \mathrm{dL}$. TC cervicotoraco-abdominal: conglomerados adenopáticos en todas las cadenas ganglionares mediastínicas sin afectación en áreas infradiafragmáticas. Hepatomegalia homogénea. Gammagrafía con galio: captación patológica a nivel mediastínico sin afectación de otras áreas. Biopsia de médula ósea: ausencia de infiltración tumoral. Biopsia de adenopatía cervical derecha: enfermedad de Hodgkin tipo esclerosis nodular grado II.

Se instauró tratamiento poliquimioterápico según pauta ABVD. En el momento actual, dos años desde el diagnóstico, la paciente se encuentra en remisión completa. Durante este tiempo la paciente no ha tenido nuevos brotes de lesiones cutáneas.

Las lesiones cutáneas asociadas a la EH se presentan entre el 17 y el 53\% de los casos (1). Estas lesiones se clasifican en especificas, inespecíficas y una forma exclusivamente cutánea de $\mathrm{EH}$ (1). La paciente había tenido un cuadro cutáneo abigarrado de dos años de evolución antes de ser diagnosticada de EH. Este proceso cutáneo fue diagnosticado de vasculitis leucocitoclástica.

La VL o vasculitis por hipersensibilidad afecta a arterias de pequeño calibre, arteriolas, capilares y vénulas postcapilares fundamentalmente de la piel. Se observa un infiltrado inflamatorio neutrofílico y fragmentos nucleares de granulocitos (leucocitoclastia).

Tabla I

PACIENTES DESCRITOS CON VASCULITIS LEUCOCITOCLÁSTICA ASOCIADA A EH

\begin{tabular}{llll}
\hline Referencia & Sexo / edad & Relación temporal con la EH & \\
\hline Sams et al, 1968 (3) & Varón / 53 años & 2 meses antes de EH & Granuloma renal \\
Lonchampt et al, 1984 (4) & Mujer / 42 años & Simultánea & Estadio III A \\
Lonchampt et al, 1984 (4) & Varón / 23 años & Varios meses antes de EH & Estadio III B \\
Kesseler y Slater, 1986 (5) & Varón / 72 años & 4 semanas antes que EH & Estadio II B, tipo deplección linfocítica \\
Kesseler y Slater, 1986 (5) & Varón / 61 años & Simultánea & Estadio II, tipo celularidad mixta \\
Beylot et al, 1989 (6) & Mujer / 55 años & 11 años tras EH & No especificado \\
Cransac et al, 1993 (2) & Varón / 46 años & 3 meses antes de EH & Estadio II \\
Cransac et al, 1993 (2) & Varón / 69 años & 12 meses antes de EH & Estadio II \\
Presente caso & Mujer / 53 años & 18 meses antes de EH & Estadio II A, tipo esclerosis nodular \\
\hline
\end{tabular}


La forma de presentación típica es la púrpura palpable. Se postula que el origen de esta vasculitis es autoinmune. Aunque el antígeno promotor suele ser desconocido, se ha asociado a múltiples entidades (infecciones víricas y bacterianas, fármacos, conectivopatías y neoplasias). Los tumores hematológicos suponen entre el 3 y el $12 \%$ de las causas de VL (2). Aunque se describe con mayor frecuencia en tumores de la serie mieloide, la VL también se ha asociado a la EH. Hemos encontrado 8 casos de VL asociada a EH (2-6) (Tabla I). La mediana de edad de aparición fue de 53 años, con una relación hombre / mujer de 2:1. En 5 de los casos el estudio de extensión mostró un estadio II. La VL precedió entre 2 y 18 meses al linfoma en 6 casos, apareció de forma simultánea en 2 pacientes y tan solo en un caso el linfoma precedió al diagnostico de VL.

El SVCS es una forma muy poco frecuente de presentación de la EH. En una serie de 125 casos de SVCS tan solo un caso resultó ser secundario a EH (7). No hemos encontrado ninguna serie de pacientes con EH en la que se analice el SVCS como forma de comienzo.

La relación entre vasculitis leucocitoclástica y enfermedad de Hodgkin no resulta aparente. Sin embargo, el presente caso ilustra la necesidad de realizar un seguimiento cercano de cuadros vasculíticos no bien definidos y de evolución tórpida que pueden ser precursores de procesos neoplásicos. Este hecho enfatiza la conveniencia de un control evolutivo de estos pacientes durante un tiempo que resulta difícil de precisar por la escasez de referencias previas pero que, de acuerdo con esta experiencia, se podría establecer al menos en dos años.

\section{Mora Rillo, J.I. Bernardino de la Serna, T. Cobo Ibáñez, B. García García, A. Gil Aguado, J. García Puig}

Servicio de Medicina Interna. Hospital Universitario La Paz. Madrid

1. Hayes TG, Rabin VR, Rosen T, Zubler MA. Hodgkin's disease presenting in the skin: Case report and review of the literature. J Am Acad Dermatol 1990; 22: 944-7.

2. Cransac M, Vidal E, Liozon E, Lavignac C, Remenieras L, Bordessoule D. Hodgkin's disease revealed by cutaneous vasculitis: two cases. Eur J Haematol 1993; 50: 53-4.

3. Sams WM, Harville DD, Winkelmann RK. Necrotising vasculitis associated with lethal reticuloendothelial diseases. Br J Derm 1968; 80: 55560 .

4. Lonchampt F, Blanc D, Kienzler JL, Flesch M, Humbert P, Agache P. Vascularities cutanées révélatrices de lymphomes: place de la lymphadénopathie angioimmunoblastique. Sem Hôp Paris 1984; 60: 3193-4.

5. Kesseler ME, Slater DN. Cutaneous vasculitis: a presenting feature in Hodgkin's disease. J R Soc Med 1986; 79: 485-6.

6. Beylot J, Malou M, Doutre MS et al. Vascularites leucocytoclasiques et hémopathies malignes (12 observations). Rev Med Interne 1989; 10: 509-14.

7. Abner A. Approach to the patient who presents with superior vena cava obstruction. Chest 1993; 103: 394S-7S.

\section{Trombosis venosa profunda y riesgo de neoplasia}

\section{Sr. Director:}

Hemos leído con interés el artículo "Trombosis venosa profunda y cancer: actitud diagnóstica" publicado en su revista por Rodríguez y col (1). La relación entre la enfermedad tromboem- bólica y los tumores malignos es conocida desde hace mas de un siglo. La prevalencia de cáncer en pacientes que presentan enfermedad tromboembólica varía en los diferentes estudios. En nuestro medio, nosotros investigamos de manera retrospectiva la asociación entre ambas patologías durante los años 1992-1999. Nuestros objetivos fueron analizar el porcentaje de pacientes que desarrollaban una enfermedad neoplásica tras un episodio de trombosis venosa profunda (TVP), el periodo de tiempo entre ambos eventos y la localización tumoral. Se excluyeron los diagnósticos de neoplasia previos al evento tromboembólico.

Los criterios utilizados para el diagnostico de las entidades nosológicas fueron los de la CIE-9. Un total de 1.260 pacientes tuvieron al menos un episodio de TVP, de ellos a 112 se les diagnosticó una neoplasia $(8,9 \%)$. La edad media de presentación fue de 70,9 años (rango 41-90 años), el 65,2\% fueron varones. En el mismo ingreso se objetivó de forma simultanea el diagnóstico de TVP y cáncer en el 5,7\%. En el 80\% de los casos la aparición del tumor ocurrió en los 3 primeros meses tras el episodio de TVP. Los tumores mas frecuentes por orden decreciente fueron próstata, pulmón y colon entre los varones y ginecológico, mama y páncreas en las mujeres.

La incidencia de aparición de tumores tras la trombosis venosa varía en función de las series $(7,6-17 \%)(2,3)$. De nuestro estudio, al igual que ha sido sugerido por otros autores en pacientes menores de 40 años con un episodio de TVP primario no existe un mayor riesgo de cáncer (4). Los tumores que aparecieron en nuestros pacientes son similares a los publicados en la literatura $(5,6)$. Sin embargo, a partir de dicha edad, existe un riesgo 4 veces superior de presentar neoplasias sólidas ocultas durante el primer año (7). A pesar de ello, no está justificada la búsqueda sistemática de un tumor en todos los pacientes con TVP $(5,6)$. No obstante, algunos autores son partidarios de realizar una investigación más extensa al objeto de detectar de forma precoz un tumor ante la posibilidad de mejoría de su pronostico $(8,9)$. Parece recomendable una vigilancia activa de los pacientes sobre todo en los 6 primeros meses tras la TVP dado que la mayoría de las neoplasias aparecen en dicho intervalo de tiempo.

\section{Zabalza Azparren, J. D. Mosquera Lozano, A. Arizti Martín, M. Vallejo García}

Servicio de Medicina Interna. Complejo Hospitalario San Millán-San Pedro. Logroño

1. Rodríguez Blanco A, Rodríguez Barrientos R, Estelles Piera F. Trombosis venosa profunda y cancer: actitud diagnostica. An Med Interna (Madrid) 2002; 19: 657-8.

2. Prandoni P, Lensing AWA, Búller HR, Cogo A, Prins MH, Cattelan AM, et al. Deep vein thrombosis and the incidence of subsequent symptomatic malignant disease. N Engl J Med 1992; 327: 1128-33.

3. Monreal M, Lafoz E, Casals A, et al. Occult cancer in patients with venous thrombosis: a systematic approach. Cancer. 1991: 67: 541-5.

4. Subira M, Mateo J, Souto JC, Altes A, Fontcuberta J. Lack of association between venous thrombosis and subsequent malignancy in a retrospective cohort study in young patients. Am J Hematol 1999;60: 181-4.

5. Sorensen HT, Mellemkajaer L, Steffensen FH, Olsen JH, Nielsen GL The risk of a diagnosis of cancer after primary deep venous thrombosis or pulmonary embolism. N Engl J Med 1998; 338: 1169-73.

6. Monreal M, Prandoni P. Venous thromboembolism as first manifestation of cancer. Semin Thromb Hemost 1999; 25: 131-6.

7. Barón JA, Gridley G, Weiderpass E, Nyren O, Linet M. Venous thromboembolism and cancer. Lancet 1998; 351: 1077-80.

8. Piccioli A, Prandomi P. Idiopathic venous thromboembolism as a first manifestation of cancer. Haemostasis 2001; 31: 37-9.

9. Prandoni P. Cancer and thromboembolic disease; how important is the risk of thrombosis? Cancer Treat Rev 2002; 28: 133-6. 